\title{
Induction of the pro-inflammatory NF-kB-sensitive miRNA-146a by human neurotrophic viruses
}

\author{
James M. Hill ${ }^{1,2}$, Christian Clement ${ }^{3}$, Yuhai Zhao² and Walter J. Lukiw ${ }^{2,4 *}$ \\ Departments of Microbiology and Pharmacology, Louisiana State University Health Science Center, New Orleans, LA, USA \\ ${ }^{2}$ LSU Neuroscience Center and Department of Ophthalmology, Louisiana State University Health Science Center, New Orleans, LA, USA \\ ${ }^{3}$ Infectious Diseases, Experimental Therapeutics and Human Toxicology Lab, Department of Natural Sciences, Southern University at New Orleans, New Orleans, \\ LA, USA \\ ${ }^{4}$ Department of Neurology, Louisiana State University Health Science Center, New Orleans, LA, USA \\ ${ }^{*}$ Correspondence: wlukiw@lsuhsc.edu \\ Edited by: \\ Reena Ghildyal, University of Canberra, Australia \\ Reviewed by: \\ Arup Banerjee, Translational Health Science and Technology Institute (THSTI), India \\ Elena Gazina, The Florey Institute of Neuroscience and Mental Health, Australia
}

Keywords: Alzheimer's disease (AD), aluminum sulfate, ebola virus, innate-immune response, messenger RNA (mRNA) and microRNA (miRNA), microRNA-146a, neuroinflammation, prion disease

A remarkably wide variety of human neurotrophic viruses-ranging from herpes simplex 1 (HSV-1; Herpesviridae; dsDNA genome) to Hantavirus (HTV; Bunyaviridae; (-)ssRNA genome) to human immunodeficiency virus (HIV; Retroviridae; (+)ssRNA genome) are associated with the rapid up-regulation of the NF-kB-sensitive pro-inflammatory microRNA-146a (miRNA-146a) in the host shortly after infection. This significant miRNA-146a up-regulation appears to be beneficial to the infecting virus as part of an immune-evasion strategy. Interestingly, miRNA-146a is also significantly up-regulated in several human central nervous system (CNS) disorders. These include Alzheimer's disease (AD) and prion disease where miRNA146a participates in pro-inflammatory and innate-immune signaling. This opinion paper will comment on some recently clarified roles for the NF-kBregulated, pro-inflammatory miRNA-146a in viral-induced cellular dysfunction, and how anti-miRNA-146a and/or related therapeutic strategies may be beneficial in the clinical management of a broad spectrum of viral-mediated CNS disease.

The 22 nucleotide, non-coding, single stranded RNA (ssRNA) miRNA-146a (5'-UGAGAACUGAAUUCCAUGGGUU3'; 41\% C+G; NR_029701) lies at the crossroads of multiple biological processes involved in the innate-immune response, viral-infection and inflammatory disease (Lukiw and Pogue, 2007; Cui et al., 2010; Lukiw, 2012; Saba et al., 2014). miRNA-146a, encoded at chromosome 5q33.3 (chr 5q33.3) in humans, is a rapidly induced, NF-kB-sensitive pro-inflammatory miRNA with a relatively short half-life of about $\sim 2 \mathrm{~h}$ in the human CNS (Taganov et al., 2006; Lukiw et al., 2008; Sethi and Lukiw, 2009; Li et al., 2010; Kroesen et al., 2015). Initially described as being significantly up-regulated after microbial lipopolysaccharide (LPS) stimulation of monocytes and under transcriptional control by NF- $\kappa$, miRNA-146a was subsequently found to be: (i) up-regulated by proinflammatory cytokines (such as IL- $1 \beta$ and TNF $\alpha$; Taganov et al., 2006; Lukiw et al., 2008; Cui et al., 2010); (ii) induced by metal sulfate-generated reactive oxygen species (ROS; Pogue et al., 2009); (iii) up-regulated by neurotoxic 42 amino acid amyloid beta (A $\beta 42)$ peptides in human primary brain cells (Li et al., 2010; Alexandrov et al., 2011); and (iv) implicated as a key regulator of innate-immune signaling in part through interleukin receptor-associated kinase (IRAK) activation (Cui et al., 2010; Saba et al., 2014). Subsequent sequencing across the chr 5 q33.3 locus indicated the presence of 3 tandem, canonical NF-kB-binding sites in the $5^{\prime}$ regulatory-region of the miRNA146a gene (Sethi and Lukiw, 2009; Cui et al., 2010). Combined with functionality and NF-kB-inhibition assays miRNA-

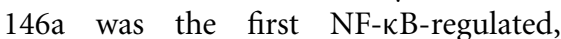
pro-inflammatory miRNA identified in the human CNS (Li et al., 2010, 2011). The most significant miRNA-146a abundances to date have been found in astroglial and microglial cells, the later representing the "resident scavenging-macrophages" of the CNS, and key participants in the brain's innate-immune surveillance and inflammatory response-systems ( $\mathrm{Li}$ et al., 2010, 2011; Saba et al., 2012). While only basally expressed in the CNS, miRNA146 a can be induced 2- to 25 -fold or higher in cultured human primary brain cells after the application of several different classes of physiological stressors including treatment with (i) neurotropic virus (Hill et al., 2009; Lukiw et al., 2009; Li et al., 2010); (ii) neurotoxic metal sulfates (such as aluminum sulfate at low nanomolar concentrations; Pogue et al., 2009); (iii) microbial endotoxins including LPS (Taganov et al., 2006); and (iv) pro-inflammatory cytokines and $\mathrm{A} \beta$ peptides, either alone or in combination (Taganov et al., 2006; Lukiw et al., 2008, 2010; Li et al., 2010). While the mechanism for miRNA-146a-mediated immune-evasion is still not fully understood, in humans this process appears to require the activation of NF-kB; other transcription factors such as AP1 may be used in miRNA-146a activation in mice (Tung et al., 2010; Ho et al., 2014; Wang et al., 2014). 
A surprisingly large number of different types of potentially incapacitating or lethal viruses, possessing either DNA or RNA genomes, have been shown to significantly induce miRNA-146a in the human CNS, immune, lymphatic, hepatic or circulatory systems, and these include (alphabetically-ordered): (i) Chikungunya virus (CHIKV; Togaviridae; (+)ssRNA genome; Selvamani et al., 2014); (ii) enterovirus 71 (EV71; Picornaviridae; (+)ssRNA genome; Ho et al., 2014); (iii) Epstein-Barr virus (EBV; Herpesviridae; dsDNA genome; Jonigk et al., 2013); (iv) Hantavirus (HTV; Bunyaviridae; (-)ssRNA genome; Shin et al., 2013); (v) hepatitis C virus (HCV; Flaviviridae; (+)ssRNA genome; Joshi et al., 2013); (vi) herpes simplex virus1 (HSV-1; Herpesviridae; dsDNA genome; Higaki et al., 2003; Hill et al., 2009; Lukiw et al., 2010); (vii) Henipavirus (Hendra) virus (HeV; Paramyxoviridae; (-)ssRNA genome; Stewart et al., 2013); (viii) human influenza A viruses (H1N1/H3N2; Orthomyxoviridae; (+)ssRNA genome; Chen et al., 2012; Terrier et al., 2013); (ix) hepatitis B virus (HBV; Hepadnaviridae; dsDNA genome; Liu et al., 2009); (x) human immunodeficiency virus (HIV; Retroviridae; (+)ssRNA genome; Duskova et al., 2013; (xi) human T-cell leukemia (lymphotropic) virus type 1 (HTLV1; Retroviridae; (+)ssRNA genome; Pichler et al., 2008); and (xii) Japanese encephalitis virus (JEV; Flaviviridae; (+)ssRNA genome; Pareek et al., 2014). Note that (i) this viral-miRNA-146ainduction/association are all relatively recent discoveries with more than three quarters identified within the last 22 months; (ii) all of the most recent viralhost miRNA-nucleoplasmic signaling studies indicate the up-regulation of miRNA-146a; and (iii) viral infection involving each virus mentioned above is associated with progressive neuropathological change. Interestingly (i) miRNA146a up-regulation has been associated with common age-related, human inflammatory degenerations such as sporadic Alzheimer's disease $(\mathrm{AD})$, and the rare sporadic prion diseases Creutzfeldt-Jakob disease (sCJD) and Gerstmann-StrausslerScheinker (GSS) syndrome; and (ii) the etiopathogenesis of $\mathrm{AD}$ has recently been associated with multiple viral infections, and most recently with latent $\mathrm{HCV}, \mathrm{HIV}-1$ or HSV-1 reactivation (Hill et al., 2009, 2014; Lukiw et al., 2011; Ball et al., 2013; Alexandrov et al., 2014).

Under suitable physiological conditions, often within minutes after viral infection, signaling via the pre-existing, heterodimeric transcription factor NF$\mathrm{kB}$ is accomplished by complex, highly interdependent, viral-mediated regulatory mechanisms. These involve proteinprotein interactions, phosphorylation, ADP-ribosylation, nucleocytoplasmictrans-location, ubiquitination and proteolytic-degradation (Vallabhapurapu and Karin, 2009; Cui et al., 2010; Lee and Covert, 2010; Yarbrough et al., 2014; Di Girolamo, 2015). The most ubiquitous NF- $\kappa \mathrm{B}$ members in non-stimulated cell cytoplasm are the p50 and p65 (RelA) subunits forming the heterotypic p50/p65 NF-kB dimer complexed with members of the IкB-inhibitor family (which prevents nuclear translocation; Zanella et al., 2013; Di Girolamo, 2015). Typically, after viral-mediated phosphorylation of I $\mathrm{B} \alpha$ at specific serine residues, Iк $\mathrm{B} \alpha$ dissociates from the p50/p65 dimer, is ubiquitinated and degraded by the proteasome, allowing the majority of NF- $\kappa \mathrm{B}$ complexes to translocate through the nuclear pore complex (NPC; typically 10,000 nuclear pores/neuron; Threadgold, 1976). Here NF-kB subsequently recognizes genomic NF-kB binding sites in target gene regulatory regions, to transiently activate RNA Pol II-mediated transcription (Vallabhapurapu and Karin, 2009; Cui et al., 2010; Zanella et al., 2013; Di Girolamo, 2015). The miRNA-146a gene for example may be up-regulated 10 -fold or more within minutes of viral infection; importantly NF- $\mathrm{B}$ activation is usually terminated via $I \kappa \mathrm{B}$ protein re-synthesis and NF-kB-re-inhibition (Schmid and Birbach, 2008; Hill et al., 2009, 2014; Cui et al., 2010; Lukiw et al., 2010). Gel shift assays and livecell fluorescence microscopy indicate that NF- $\kappa \mathrm{B}$ activation may exhibit oscillatory patterns, with levels of nuclear NF- $\kappa \mathrm{B}$ alternately increasing-and-decreasing; this suggests the intriguing possibility that NF-kB-based signaling might exploit the timing of protein-modification and nucleocytoplasmic shuttling to regulate gene expression (Spiller et al., 2010;
Kodaman et al., 2014). Oscillatory variation in miRNA-146a abundance is not well understood, indeed viral-mediated phosphorylation of $\mathrm{IkB}$ and NF-kB activation and nucleocytoplasmic trafficking is complicated as different viruses may recruit different viral or host proteins to target different signaling components of the NF-kB pathway using multiple strategies. For example polyubiquitination of the $(+)$ ssRNA HTVL-1 virus encoded Tax protein activates $\mathrm{IkB}$ kinase resulting in NF-kB activation and nucleocytoplasmic translocation, while the dsDNA EBV-encoded latent membrane protein 1 (LMP1) not only activates $\mathrm{IkB}$ kinase to induce nucleocytoplasmic trafficking of NF-kB but also appears to be involved in additional mechanisms including LMP1mediated interaction with nuclear proteins (Currer et al., 2012; Ersing et al., 2013). Interestingly, many neurotrophic viruses inhibit nucleocytoplasmic trafficking of host mRNAs to promote cytoplasmic viral replication and disrupt expression of antiviral factors by the host (Yarbrough et al., 2014). What is remarkable is that despite a tremendous variation in their biophysical and genomic structure, nucleic acid type, size and life-cycle, in humans all miRNA146a-inducing neurotrophic viruses appear to share the common capabilities: (i) to target NF-kB-mediated gene expression; (ii) to induce complex nuclear and/or nucleocytoplasmic signaling that processes miRNA-146a precursors to export mature miRNA-146a back into the cytoplasm; and (iii) to drive a miRNA-146a-mediated arachidonic acid signaling cascade with subsequent pro-inflammatory and pathogenic consequences (Hill et al., 2009; Lukiw et al., 2010; Alexandrov et al., 2014; Yarbrough et al., 2014).

Out of about 24,000 miRNAs so far identified in all species, only about 300 are encoded by viruses (miRBase v.20; Liu, 2014). There is evidence that viralencoded miRNAs regulate the expression of their own genes or the host's genes, or both (Liu, 2014; Yao and Nair, 2014). dsDNA viruses encode most of the viralencoded miRNAs, with members of the family Herpesviridae accounting for the vast majority, indicating the significance of viral miRNA-mediated gene regulation in the biology of HSV infection (see above; Yao and Nair, 2014). In general 
DNA viruses that contain miRNA encoded in their viral DNA require access to the RNA polymerase II and miRNA processing machinery located within the nucleus in order to express that miRNA. In contrast, RNA viruses can replicate in the cytoplasm and, therefore, rarely encode miRNA (Liu, 2014; Swaminathan et al., 2014; Yao and Nair, 2014). There are, however, notable exceptions - for example infection with Ebola virus [EBOV; Filoviridae; (-)ssRNA genome] that causes a highly lethal hemorrhagic fever syndrome in humans rapidly induces 3 EBOV genome-derived miRNAs that subsequently target host mRNA (Liang et al., 2014). Indeed, perhaps as part of complex survival and immune-evasion strategies, neurotrophic viruses may modulate host miRNA precursor processing to favor viral miRNA production, thus contributing to viral-disease pathogenesis via multiple and highly interactive mechanisms (Conrad and Niepmann, 2014; Liu, 2014; Yao and Nair, 2014; Yarbrough et al., 2014).

In summary, viruses have evolved multiple and complex strategies to subvert and evade the host immune-response to ensure their own replication and survival (Hill et al., 2014; Kodaman et al., 2014; Yarbrough et al., 2014). While there is still debate as to whether up-regulated miRNA-146a is beneficial to the infecting virus or a protective host innate-immune response, at least 7 recent observations suggest that a virally-induced NF-kBmediated up-regulation of miRNA-146a is significantly pathogenic and disruptive to homeostatic CNS function: (i) the antiviral acycloguanosine acyclovir prevents an HSV-1-induced miRNA-146a-activated pro-inflammatory cell-death program in human CNS cells via reduction in miRNA-146a abundance (Lukiw et al., 2010); (ii) up-regulated miRNA-146a has been shown to significantly down-regulate expression of complement factor- $\mathrm{H}$ to induce a progressive and lethal proinflammatory degeneration in stressed human primary brain cells (Cui et al., 2010; Alexandrov et al., 2014); (iii) both viral and cytokine (IL-1 $\beta$, TNF $\alpha$ ) induced up-regulation of miRNA-146a triggers a chronic human retinal-degeneration (Kutty et al., 2013; Alexandrov et al., 2014; Hill et al., 2014); (iv) a progressive upregulation of miRNA-146a accompanies pro-inflammatory neuropathology in lethal human CNS disorders including sporadic $\mathrm{AD}$ and the human-prion diseases GSS and sCJD (Lukiw et al., 2011; Saba et al., 2012); (v) a progressive upregulation of miRNA-146a accompanies AD-type neuropathology in several transgenic animal models of $\mathrm{AD}$ (including Tg2576 and 5xFAD; Alexandrov et al., 2011, 2014; Li et al., 2011); (vi) quenching of miRNA-146a using anti-miRNA-146a strategies restores homeostatic immune signaling in CHIKV-infected human fibroblasts (Selvamani et al., 2014); and (vii) inhibition of EV71-induced miRNA-146a-upregulation employing anti-miRNA-146a strategies has been observed to inhibit viral propagation and improve survival rates in mouse models (Ho et al., 2014). It is our opinion: (i) that NF-kB inhibition may not be an effective therapeutic strategy for neurotrophic viral infections because NF-kB is a ubiquitous transcription factor with large potential for off-target effects; and (ii) that virallyinduced miRNA-146a excess could be effectively neutralized using perfectly complementary locked nucleic acid-stabilized anti-miRNA-146a oligonucleotides, and thereby act as an anti-viral agent for a wide variety of DNA- and RNA-virus-induced disease (Lukiw, 2013; Maguire et al., 2014). Indeed, a major advancement in antiviral therapy might involve a broad-spectrum, anti-miRNA-146a strategy which, perhaps in combination with antivirals such as acyclovir and/or the recently described gene editing methods using CRISPR/Cas9 (clustered regularly interspaced short palindromic repeats/caspase 9)-mediated or other gene therapy technologies (Doudna and Charpentier, 2014; Maguire et al., 2014; Hochstrasser and Doudna, 2015). We envision these to have considerable therapeutic potential in the future clinical management of viral infections where miRNA-146a up-regulation appears to play a pathogenic role.

\section{ACKNOWLEDGMENTS}

This research was presented in part at the Society for Neuroscience (SFN) 44th Annual Meeting, Washington DC, USA, 14-19 November 2014. Research in the Lukiw laboratory on microRNA, non-coding single stranded RNA (ssRNA), the innate-immune response, amyloidogenesis, and neuroinflammation in $\mathrm{AD}$, retinal and prion disease using post-mortem brain tissues, was supported through a COBRE III Pilot Award, an unrestricted grant from Research to Prevent Blindness (RPB), the Louisiana Biotechnology Research Network (LBRN), and NIH grants NEI EY006311 and NIA AG038834.

\section{REFERENCES}

Alexandrov, P. N., Dua, P., and Lukiw, W. J. (2014) Up-Regulation of miRNA-146a in progressive, age-related inflammatory neurodegenerative disorders of the human CNS. Front. Neurol. 5:181. doi: 10.3389/fneur.2014.00181

Alexandrov, P. N., Pogue, A., Bhattacharjee, S., and Lukiw, W. J. (2011). Retinal amyloid peptides and complement factor $\mathrm{H}$ in transgenic models of Alzheimer's disease. Neuroreport 2, 623-627. doi: 10.1097/WNR.0b013e3283497334

Ball, M. J., Lukiw, W. J., Kammerman, E. M., and Hill, J. M. (2013). Intracerebral propagation of Alzheimer's disease: strengthening evidence of a herpes simplex virus etiology. Alzheimers Dement. 9, 169-175. doi: 10.1016/j.jalz.2012. 07.005

Chen, J., Yuan, L., Fan, Q., Su, F., Chen, Y., and Hu, S. (2012). Adjuvant effect of docetaxel on the immune responses to influenza A H1N1 vaccine in mice. BMC Immunol. 13:36. doi: 10.1186/14712172-13-36

Conrad, K. D., and Niepmann, M. (2014). The role of microRNAs in hepatitis $\mathrm{C}$ virus RNA replication. Arch. Virol. 159, 849-862. doi: 10.1007/s00705013-1883-4

Cui, J. G., Li, Y. Y., Zhao, Y., Bhattacharjee, S., and Lukiw, W. J. (2010). Differential regulation of interleukin-1 receptor-associated kinase-1 (IRAK1) and IRAK- 2 by miRNA-146a and NF-kB in stressed human astroglial cells and in Alzheimer disease. J. Biol. Chem. 285, 38951-38960. doi: 10.1074/jbc.M110.178848

Currer, R., Van Duyne, R., Jaworski, E., Guendel, I., Sampey, G., Das, R., et al. (2012). HTLV tax: a fascinating multifunctional co-regulator of viral and cellular pathways. Front. Microbiol. 3:406. doi: 10.3389/fmicb.2012.00406

Di Girolamo, M. (2015). Regulation of nucleocytoplasmic transport by ADP-ribosylation: the emerging role of karyopherin- $\beta 1$ MonoADP-Ribosylation by ARTD15. Curr. Top. Microbiol. Immunol. 384, 189-209. doi: 10.1007/82_2014_421

Doudna, J. A., and Charpentier, E. (2014). Genome editing. The new frontier of genome engineering with CRISPR-Cas9. Science 346:1258096. doi: 10.1126/science.1258096

Duskova, K., Nagilla, P., Le, H. S., Iyer, P., Thalamuthu, A., Martinson, J., et al. (2013). MicroRNA regulation and its effects on cellular transcriptome in human immunodeficiency virus-1 (HIV-1) infected individuals with distinct viral load and CD4 cell counts. BMC Infect Dis. 13:250. doi: 10.1186/1471-2334-13-250

Ersing, I., Bernhardt, K., and Gewurz, B. E. (2013). NF-кB and IRF7 pathway activation 
by Epstein-Barr virus latent membrane protein 1 . Viruses. 5, 1587-1606. doi: 10.3390/v5061587

Higaki, S., Gebhardt, B., Lukiw, W. J., Thompson, H., and Hill, J. (2003). Gene expression profiling in the HSV-1 latently infected mouse trigeminal ganglia following hyperthermic stress. Curr. Eye Res. 26, 231-238. doi: 10.1076/ceyr.26.3.231.14892

Hill, J. M., Clement, C., Pogue, A. I., Bhattacharjee, S., Zhao, Y., and Lukiw, W. J. (2014). Pathogenic microbes, the microbiome, and Alzheimer's disease (AD). Front. Aging Neurosci. 6:127. doi: 10.3389/fnagi.2014.00127

Hill, J. M., Zhao, Y., Clement, C., Neumann, D. M., and Lukiw, W. J. (2009). HSV-1 infection of human brain cells induces miRNA-146a and Alzheimertype inflammatory signaling. Neuroreport 20, 1500-1505. doi: 10.1097/WNR.0b013e32833 $29 \mathrm{c} 05$

Ho, B. C., Yu, I. S., Lu, L. F., Rudensky, A., Chen, H. Y., Tsai, C. W., et al. (2014). Inhibition of miR-146a prevents enterovirus-induced death by restoring the production of type I interferon. Nat. Commun. 5:3344. doi: $10.1038 /$ ncomms 4344

Hochstrasser, M. L., and Doudna, J. A. (2015). Cutting it close: CRISPR-associated endoribonuclease structure and function. Trends Biochem. Sci. 40, 58-66. doi: 10.1016/j.tibs.2014. 10.007

Jonigk, D., Izykowski, N., Maegel, L., Schormann, E., Maecker-Kolhoff, B., Laenger, F., et al. (2013). MicroRNA expression in Epstein-Barr virus-associated post-transplant smooth muscle tumours is related to leiomyomatous phenotype. Clin. Sarcoma Res. 3:9. doi: 10.1186/20453329-3-9

Joshi, D., Salehi, S., Brereton, H., Arno, M., Quaglia, A., Heaton, N., et al. (2013). Distinct microRNA profiles are associated with the severity of hepatitis C virus recurrence. Liver Transpl. 19, 383-394. doi: $10.1002 /$ lt.23613

Kodaman, N., Sobota, R. S., Mera, R., Schneider, B. G., and Williams, S. M. (2014). Disrupted human-pathogen co-evolution: a model for disease. Front. Genet. 5:290. doi: 10.3389/fgene.2014. 00290

Kroesen, B. J., Teteloshvili, N., Smigielska-Czepiel, K., Brouwer, E., Boots, A. M., van den Berg, A., et al. (2015). Immuno-miRs: critical regulators of T-cell development, function and ageing. Immunology 144, 1-10. doi: 10.1111/imm. 12367

Kutty, R. K., Nagineni, C. N., Samuel, W., Vijayasarathy, C., Jaworski, C., Duncan, T., et al. (2013). Differential regulation of microRNA146a and microRNA-146b-5p in human retinal pigment epithelial cells by interleukin- $1 \beta$, tumor necrosis factor- $\alpha$, and interferon- $\gamma$. Mol. Vis. 19 , 737-750.

Lee, T. K., and Covert, M. W. (2010). Highthroughput, single-cell NF-kB dynamics. Curr. Opin. Genet. Dev. 20, 677-683. doi: 10.1016/j.gde.2010.08.005

Li, L., Chen, X. P., and Li, Y. J. (2010). MicroRNA146a and human disease. Scand. J. Immunol. 71, 227-231. doi: 10.1111/j.1365-3083.2010.02383.x

Li, Y. Y., Cui, J. G., Dua, P., Pogue, A. I., Bhattacharjee, S., and Lukiw, W. J. (2011). Differential expression of miRNA-146a-regulated inflammatory genes in human primary neural, astroglial and microglial cells. Neurosci. Lett. 499, 109-113. doi: 10.1016/j.neulet.2011.05.044

Liang, H., Zhou, Z., Zhang, S., Zen, K., Chen, X., and Zhang, C. (2014). Identification of Ebola virus microRNAs and their putative pathological function. Sci. China Life Sci. 57, 973-981. doi: 10.1007/s11427-014-4759-2

Liu, D. G. (2014). MicroRNAs in human virus genomes: helping hands for viral Infection. Microrna 3, 75-85.

Liu, Y., Zhao, J. J., Wang, C. M., Li, M. Y., Han, P., Wang, L., et al. (2009). Altered expression profiles of microRNAs in a stable hepatitis B virusexpressing cell line. Chin. Med. J. (Engl). 122, 10-14. doi: 10.3901/JME.2009.11.010

Lukiw, W. J. (2012). NF-kB-regulated micro RNAs (miRNAs) in primary human brain cells. Exp. Neurol. 235, 484-490. doi: 10.1016/j.expneurol.2011.11.022

Lukiw, W. J. (2013). Antagonism of NF-кB-upregulated micro RNAs (miRNAs) in sporadic Alzheimer's disease (AD)-anti-NF- $\mathrm{KB}$ vs. antimiRNA strategies. Front. Genet. 4:77. doi: 10.3389/fgene. 2013.00077

Lukiw, W. J., Cui, J. G., Li, Y. Y., and Culicchia, F. (2009). Up-regulation of micro-RNA221 (miRNA-221; chr Xp11.3) and caspase-3 accompanies down-regulation of the survivin-1 homolog BIRC1 (NAIP) in glioblastoma multiforme (GBM). J. Neurooncol. 91, 27-32. doi: 10.1007/s11060-008-9688-0

Lukiw, W. J., Cui, J. G., Yuan, L. Y., Bhattacharjee, P. S., Corkern, M., Clement, C., et al. (2010). Acyclovir or A $\beta 42$ peptides attenuate HSV-1-induced miRNA-146a levels in human primary brain cells. Neuroreport 21 , 922-927. doi: 10.1097/WNR.0b013e32833 da5la

Lukiw, W. J., Dua, P., Pogue, A. I., Eicken, C., and Hill, J. M. (2011). Upregulation of micro RNA-146a(miRNA-146a), a marker for inflammatory neurodegeneration, in sporadic Creutzfeldt-Jakob disease (sCJD) and Gerstmann-Straussler-Scheinker (GSS) syndrome. J. Toxicol. Environ. Health A 74, 1460-1468. doi 10.1080/15287394.2011.618973

Lukiw, W. J., and Pogue, A. I. (2007). Induction of specific miRNA species by ROS-generating metal sulfates in primary human brain cells. J. Inorg. Biochem. 101, 1265-1269. doi: 10.1016/j.jinorgbio.2007.06.004

Lukiw, W. J., Zhao, Y., and Cui, J. G. (2008). An NF-kB-sensitive miRNA-146a-mediated inflammatory circuit in Alzheimer disease and in stressed human brain cells. J. Biol. Chem. 283, 31315-31322. doi: 10.1074/jbc.M80 5371200

Maguire, C. A., Ramirez, S. H., Merkel, S. F., SenaEsteves, M., and Breakefield, X. O. (2014). Gene therapy for the nervous system: challenges and new strategies. Neurotherapeutics 11, 817-839. doi: 10.1007/s13311-014-0299-5

Pareek, S., Roy, S., Kumari, B., Jain, P., Banerjee, A., and Vrati, S. (2014). MiR-155 induction in microglial cells suppresses Japanese encephalitis virus replication and negatively modulates innate immune responses. J. Neuroinflammation 11:97. doi: 10.1186/1742-2094-11-97
Pichler, K., Schneider, G., and Grassmann, R. (2008). MicroRNA miR-146a and further oncogenesis-related cellular microRNAs are dysregulated in HTLV-1-transformed T lymphocytes. Retrovirology 5:100. doi: 10.1186/1742-46 90-5-100

Pogue, A. I., Li, Y. Y., Cui, J. G., Zhao, Y., Kruck, T. P., Percy, M. E., et al. (2009). Characterization of an NF-kB-regulated, miRNA-146a-mediated down-regulation of complement factor $\mathrm{H}$ in metal-sulfate-stressed human brain cells. J. Inorg. Biochem. 103:1591-1595. doi: 10.1016/j.jinorgbio.2009.05.012

Saba, R., Gushue, S., Huzarewich, R. L., Manguiat, K., Medina, S., Robertson, C., et al. (2012). MicroRNA 146a (miR-146a) is over-expressed during prion disease and modulates the innate immune response and the microglial activation state. PLOS ONE 7:e30832. doi: 10.1371/journal.pone. 0030832

Saba, R., Sorensen, D. L., and Booth, S. A. (2014). MicroRNA-146a: a dominant, negative regulator of the innate-immune response. Front. Immunol. 5:578. doi: $10.3389 /$ fimmu. 2014.00578

Schmid, J. A., and Birbach, A. (2008). IkappaB kinase beta - a key molecule in signaling to the transcription factor NF-kB. Cytokine Growth Factor Rev. 19, 157-165. doi: 10.1016/j.cytogfr.2008.01.006

Selvamani, S. P., Mishra, R., and Singh, S. K. (2014). Chikungunya virus exploits miR-146a to regulate NF-кB pathway in human synovial fibroblasts. PLoS ONE 9:e103624. doi: 10.1371/journal.pone.0103624

Sethi, P., and Lukiw, W. J. (2009). MiRNA abundance and stability in human brain: specific alterations in Alzheimer's disease temporal lobe neocortex. Neurosci. Lett. 459, 100-104. doi: 10.1016/j.neulet.2009.04.052

Shin, O. S., Kumar, M., Yanagihara, R., and Song, J. W. (2013). Hantaviruses induce cell typeand viral species-specific host microRNA expression signatures. Virology 446, 217-224. doi: 10.1016/j.virol.2013.07.036

Spiller, D. G., Wood, C. D., Rand, D. A., and White, M. R. (2010). Measurement of single cell dynamics. Nature 465, 736-745. doi: 10.1038/nature09232

Stewart, C. R., Marsh, G. A., Jenkins, K. A., Gantier, M. P., Tizard, M. L., Middleton, D., et al. (2013). Promotion of Hendra virus replication by miRNA-146a. J. Virol. 87, 3782-3791. doi: 10.1128/JVI.01342-12

Swaminathan, G., Navas-Martín, S., and MartínGarcía, J. (2014). MiRNAs \& HIV-1 infection: antiviral activities and beyond. J. Mol. Biol. 426, 1178-1197. doi: 10.1016/j.jmb.2013.12.017

Taganov, K. D., Boldin, M. P., Chang, K. J., and Baltimore, D. (2006). NF-kB-dependent induction of microRNA-146A, an inhibitor targeted to signaling proteins of innate immune responses. Proc. Natl. Acad. Sci. U.S.A. 103, 12481-12486. doi: 10.1073/pnas.0605298103

Terrier, O., Textoris, J., Carron, C., Marcel, V., Bourdon, J. C., and Rosa-Calatrava, M. (2013). Host microRNA molecular signatures associated with human $\mathrm{H} 1 \mathrm{~N} 1$ and $\mathrm{H} 3 \mathrm{~N} 2$ influenza A viruses reveal an unanticipated antiviral activity for miRna-146a. J. Gen. Virol. 94, 985-995. doi: 10.1099/vir.0.049528-0 
Threadgold, L. T. (1976). The Ultrastructure of the Animal Cell, 2nd Edn, Chapter 3; The Ultrastructure of the Nucleus. Oxford: Pergamon International Library.

Tung, W. H., Hsieh, H. L., and Yang, C. M. (2010). Enterovirus 71 induces COX-2 expression via MAPKs, NF-kappaB, and AP-1 in SK-N-SH cells: role of PGE(2) in viral replication. Cell. Signal. 22, 234-246. doi: 10.1016/j.cellsig.2009.09.018

Vallabhapurapu, S., and Karin, M. (2009). Regulation \& function of NF-kB transcription factors in the immune system. Annu. Rev. Immunol. 27, 693-733. doi: 10.1146/annurev.immunol.021908. 132641

Wang, C., Gao, L., Jin, Y., Cardona, C. J., and Xing, Z. (2014). Regulation of host responses and viral replication by the mitogen-activated protein kinases in intestinal epithelial cells infected with Enterovirus 71. Virus Res. 197C, 75-84. doi: 10.1016/j.virusres.2014.12.016

Yao, Y., and Nair, V. (2014). Role of virusencoded microRNAs. Viruses 6, 1379-1394. doi: 10.3390/v6031379

Yarbrough, M. L., Mata, M. A., Sakthivel, R., and Fontoura, B. M. (2014). Viral subversion of nucleocytoplasmic trafficking. Traffic 15, 127-140. doi: $10.1111 /$ tra. 12137

Zanella, F., Dos Santos, N. R., and Link, W. (2013). Moving to the core: spatiotemporal analysis of Forkhead and nuclear factor- $\kappa \mathrm{B}$ nuclear translocation. Traffic 14, 247-258. doi: 10.1111/tra.12034

Conflict of Interest Statement: The authors declare that the research was conducted in the absence of any commercial or financial relationships that could be construed as a potential conflict of interest.
Received: 08 November 2014; accepted: 12 January 2015; published online: 03 February 2015.

Citation: Hill JM, Clement C, Zhao Y and Lukiw WJ (2015) Induction of the pro-inflammatory NF-kBsensitive miRNA-146a by human neurotrophic viruses. Front. Microbiol. 6:43. doi: 10.3389/fmicb.2015.00043 This article was submitted to Virology, a section of the journal Frontiers in Microbiology.

Copyright (c) 2015 Hill, Clement, Zhao and Lukiw.

This is an open-access article distributed under the terms of the Creative Commons Attribution License (CC BY). The use, distribution or reproduction in other forums is permitted, provided the original author(s) or licensor are credited and that the original publication in this journal is cited, in accordance with accepted academic practice. No use, distribution or reproduction is permitted which does not comply with these terms. 